Aktion zur Verkehrssicherheit „Risiko-Check"

— Arbeitsunfälle passieren nicht nur bei der Arbeit, sondern auch auf dem Weg zwischen Wohnung und Arbeitsstätte oder bei Fahrten im betrieblichen Auftrag. Im Jahr 2015 wurden der BGW fast 35.000 solcher Wege- und Dienstwegeunfälle gemeldet, die mehr als drei Tage Arbeitsunfähigkeit nach sich zogen. Berufsgenossenschaften, Unfallkassen und der Deutschen Verkehrssicherheitsrat (DVR) bieten in diesem Zusammenhang die Schwerpunktaktion "Risiko-Check" an. Denn wenn sich auch nicht alle Gefahrensituationen im Straßenverkehr beseitigen lassen, kann doch jeder die Risikohöhe für sich selbst beeinflussen. Drei Broschüren zu verschiedenen Verkehrsmitteln sowie eine Aktionswebsite sollen für ein stärkeres Risikobewusstsein sensibilisieren.

www.bgw-online.de

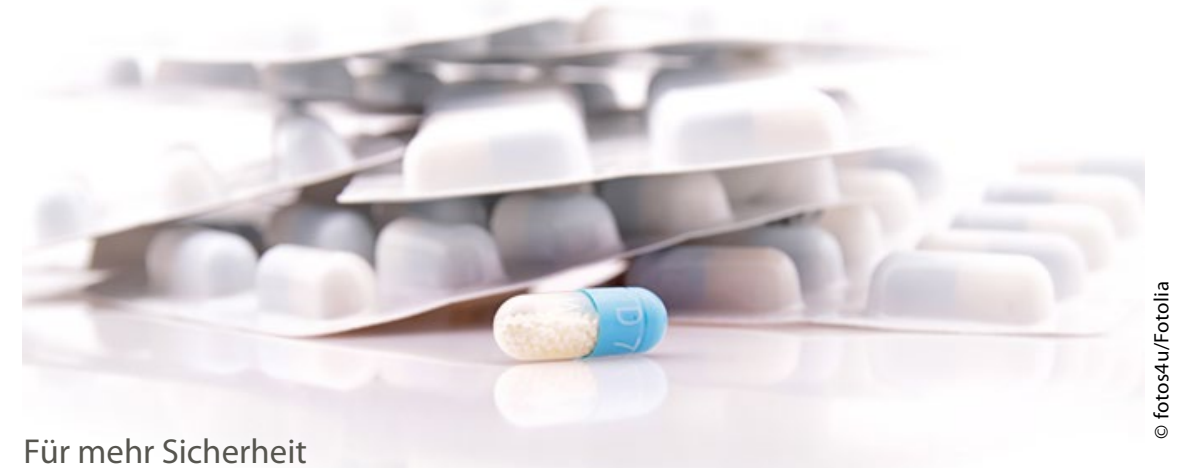

ARMIN startet Medikationsmanagement

— Die Arzneimittelinitiative Sachsen-Thüringen (ARMIN) startete im Juli 2016 das Medikationsmanagement. Durch eine koordinierte Zusammenarbeit von Arzt und Apotheker soll die Arzneimittelsicherheit für den Patienten verbessert werden. Die Arzneimittelinitiative ARMIN ist ein Modellprojekt der Kassenärztlichen Vereinigungen Sachsen und Thüringen, des Sächsischen und Thüringer Apothekerverbandes und der AOK PLUS. Das Medikationsmanagement soll vor allem chronisch kranken älteren Patienten helfen, die mehrere Medikamente einnehmen.
Arzt und Apotheker prüfen gemeinsam die verordneten und rezeptfreien Medikamente auf Wechselwirkungen sowie auf mögliche Unverträglichkeiten. Die AOK PLUS stellt zusätzlich dem betreuenden Arzt und Apotheker die Arzneimittelabrechnungsdaten der letzten sechs Monate zur Verfügung. Das Produkt dieser interdisziplinären Zusammenarbeit ist ein aktueller Medikationsplan für den Patienten.

Die Vertragslaufzeit des Modellprojekts ist bis 2018 angesetzt.

www.arzneimittelinitiative.de

\title{
Hygiene
}

\section{Ambulante Pflege kämpft mit Problemkeimen}

— Über die Hälfte der ambulanten Pflegedienste in Deutschland haben im vergangenen Jahr Menschen versorgt, die mit Problemkeimen besiedelt waren. Das geht aus einer repräsentativen Umfrage des Zentrums für Qualität in der Pflege (ZQP) unter 400 Leitungskräften hervor. Den Ergebnissen zufolge kamen ambulante Dienste am häufigsten in Kontakt mit den drei Erregergruppen MRSA, ESBL-Bildner und 3-MRGN/4MRGN. Auch der Durchfallerreger Clostridium difficile machte Pflegebedürftigen häufig zu schaffen. Unter den befragten Pflegedienstleitungen gibt zudem jeder Vierte an, dass die Mehrheit der Mitarbeiter Angst davor habe, sich mit einem Problemkeim zu infizieren. Aus Sicht der ambulanten Dienste ist fehlendes Wissen von pflegenden Angehörigen ein zentrales Problem. Doch die Pflegedienste sehen auch bei sich selbst Probleme, hygienische Standards wie die Händedesinfektion einzuhalten.

Tipp: Im Juli ist Heilberufe Spezial “Hygiene leben" erschienen. Sie können es bestellen unter www.heilberufe.de.

\section{www.zqp.de}

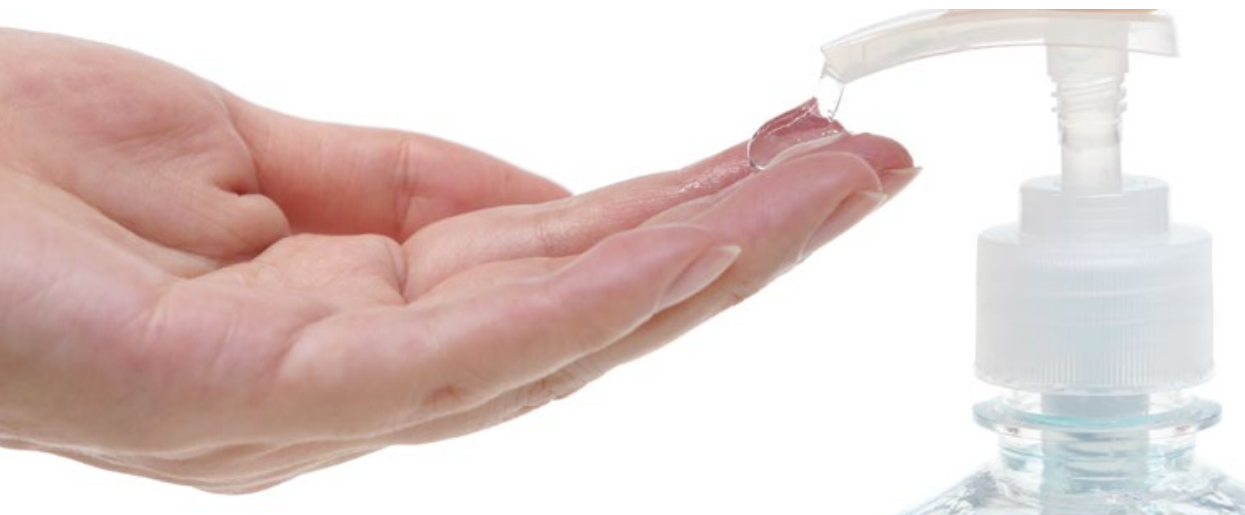

